\title{
Печатная плата - ключевой компонент, определяющий надежность конечного продукта
}

\author{
А. Чиминёв ${ }^{1}$
}

УДК 621.3.049.75| ВАК 05.27.06

\begin{abstract}
Надежность печатной платы (ПП) играет ключевую роль в правильном и бесперебойном функционировании электронного устройства. Если печатная плата выходит из строя, то устройство просто перестает работать. В данной статье мы рассмотрим пути достижения требуемой надежности печатных плат.
\end{abstract}

3 то такое надежность? Интуитивно надежность объектов связывают с недопустимостью отказов в работе. Действительно, это свойство объекта сохранять работоспособное состояние в течение необходимого времени или наработки. Иначе говоря, надежность ПП заключается в отсутствии непредвиденных недопустимых изменений ее первоначальных характеристик.

Мы выделим и рассмотрим следующие составляющие, влияющие в конечном итоге на надежность ПП: дизайн материалы, процесс изготовления, контроль и испытания, предъявляемые требования.

\section{НАДЕЖНОЕ ПРОЕКТИРОВАНИЕ}

Во многом надежность ПП закладывается на стадии проектирования. Попытка что-то исправить после того, как проект запущен в производство, может привести к огромным затратам для владельца продукции. В некоторых случаях, например в сфере производства медицинского оборудования или автомобилей, надежность ПП может стать вопросом жизни и смерти

На сегодняшний день ПП имеют чрезвычайно высокую плотность компоновки. Чем выше плотность платы, тем сложнее становится ее конструкция и процесс изготовления. По мере развития технологий нам становятся доступны всё более сложные комплексные решения. В высокотехнологичных изделиях не остается места для ошибок: их цена становится слишком высока. Именно поэтому необходимо улучшать все аспекты дизайна ПП на стадии проектирования. Совершенствование дизайна, в свою очередь, приведет к повышению надежности продукта и снижению риска возникновения неисправностей.

Применяя компоненты с малым шагом, нам приходится сужать проводники, зазоры и пояски контактных площадок с отверстиями. При изготовлении ПП это вынуждает использовать более тонкую медную фольгу для формирования проводящего рисунка. Один из наиболее

NCAB, инженер отдела качества. важных вопросов, на котором с самого начала необходимо сосредоточить внимание, - обеспечение соответствия ширины дорожек и зазоров требуемой толщине меди. Также особое внимание следует уделять параметрам переходных отверстий, поскольку они подвергаются повышенным нагрузкам при монтаже и эксплуатации ПП.

Например, при неправильном проектировании переходных отверстий недостаточная ширина пояска может привести к невозможности надежного совмещения сверловки и проводящих медных слоев. Это приводит к выходу отверстий за пределы поясков (рис. 1). Важно понимать, что если подобные отклонения находятся на внутренних слоях, то их не всегда можно обнаружить.

Другой пример - переходные отверстия, выполненные по технологии via-in-pad, то есть находящиеся прямо под контактными площадками (тип VII по стандарту IPC-4761). Такая конструкция переходных отверстий упрощает трассировку, но необходимость заполнения отверстий смолой и выполнения дополнительного цикла наращивания меди на площадках не позволяет использовать узкие проводники и зазоры, которые могли бы быть

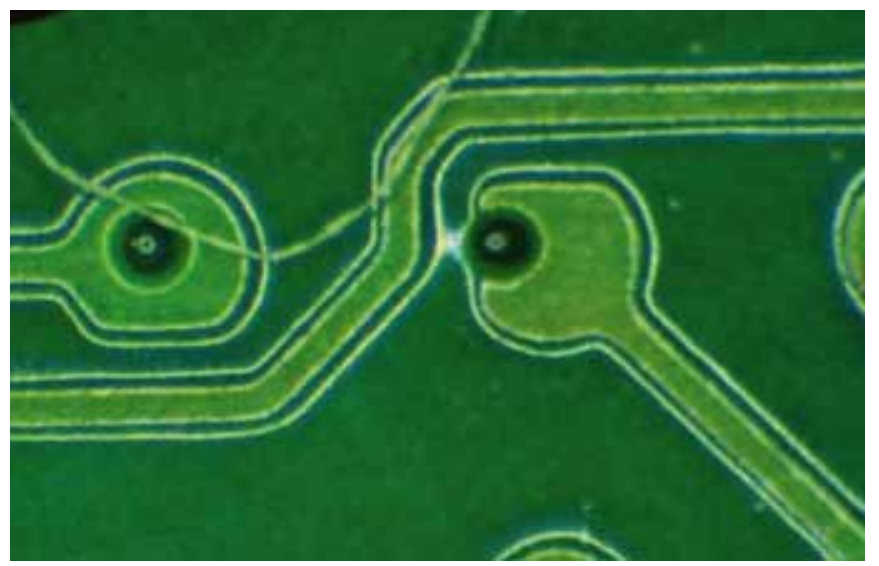

Рис. 1. Выход отверстия за пределы гарантийного пояска 


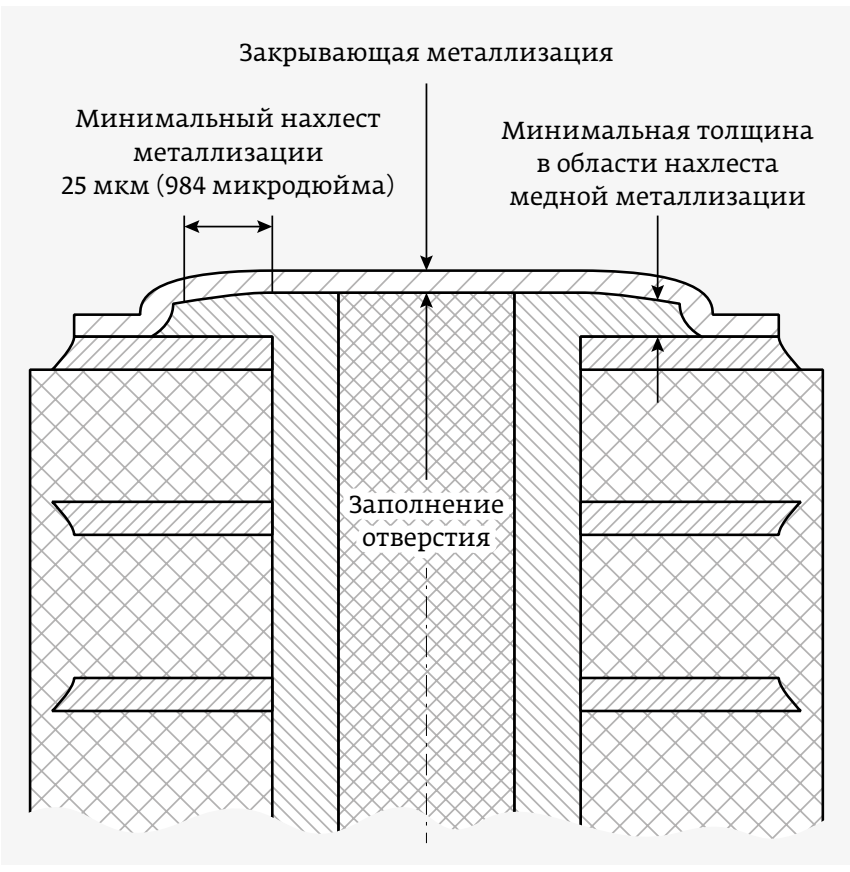

Рис. 2. Требования к нахлесту металлизации в соответствии со стандартом IPC-6012D

выполнены без использования via-in-pad. Если не принять это во внимание, чрезмерное уменьшение зазоров может потребовать отказа от нахлеста металлизации (wrap plating) на заполненных отверстиях (п. 3.6.2.11.1 стандарта IPC-6012D) (рис. 2).

Нахлест делает переходные отверстия более прочными, а электрический контакт - более надежным. Отказ от нахлеста неизбежен, если времени на коррекцию топологии уже нет. Конечно, это не значит, что контакт с отверстиями без нахлеста будет обязательно нарушен - они скорее всего выдержат монтаж, и устройства будут успешно работать. Но любые внешние воздействия, такие как вибрация, циклы нагрева и охлаждения или влажность, могут привести к тому, что электрический контакт между слоями металлизации и, как следствие, между выводом компонента и отверстием будет разорван.

Подобные риски нужно учитывать и устранять на этапе проектирования. Крайне важно, чтобы проектировщики точно знали, какие параметры достижимы при выбранной толщине меди, а также учитывали изменение этой толщины в ходе изготовления платы - тогда проблем с надежностью ПП не возникнет.

Ключом к надежной конструкции печатной платы являются стандарты серии IPC-2220, которые определяют основные принципы проектирования печатных плат в зависимости от их назначения и используемых материалов. Помимо рекомендаций IPC, необходимо также применять рекомендации по проектированию, основанные на практических возможностях изготовителей Пп.
Зачастую жесткие требования к размерам платы и высокая плотность компонентов не позволяют соблюдать все рекомендации, а высокие требования к стеку и топологии ограничивают количество подходящих производств. В таких случаях может быть оправдано применение HDI-структур, которые описаны в стандарте IPC-2226. Данные структуры содержат такие элементы, как микропереходные отверстия (microvia), которые позволят снизить плотность топологии, а следовательно, повысить надежность. В случае невозможности соблюдения всех рекомендаций отклонения должны как минимум соответствовать физическим возможностям изготовления ПП на выбранном производстве и быть согласованы с конкретным производителем.

Выполненная с учетом всех перечисленных рекомендаций печатная плата станет надежным фундаментом вашего изделия.

\section{ПРОИЗВОДСТВЕННЫЙ ПРОЦЕСС И МАТЕРИАЛЫ}

Производственный процесс может быть сколь угодно хорошо отлажен, но без наличия качественных материалов требуемая от платы надежность так и не будет достигнута, даже если обеспечен оптимальный дизайн и учтены все особенности производства. Выбор типов материалов - это часть этапа проектирования. Мы рекомендуем выбирать тип материала, отталкиваясь от классификации стандарта IPC-4101 и других документов этой серии. В стандартах IPC материалы классифицированы в зависимости от их свойств, и при заказе достаточно лишь указать условный номер спецификации на материал. Выбор конкретного бренда и марки мы рекомендуем оставлять за производителем. Этот подход оправдан, во-первых, тем, что мы не загоняем себя в узкие рамки, выбирая конкретный материал, и избегаем ситуации с задержкой сроков изготовления из-за отсутствия нужного материала на производстве и необходимостью его закупки под проект. Во-вторых, даже высококачественные материалы одного и того же типа в процессе изготовления Пп ведут себя немного по-разному. В связи с этим у каждого производства имеется свой собственный опыт максимально успешной работы с определенными брендами и марками. А значит, для достижения максимально качественного результата стоит позволить производству работать именно с теми материалами, с которыми оно уже хорошо знакомо. Выбор производства, применяющего исключительно высококачественные и зарекомендовавшие себя брендовые материалы, будет в данном случае играть решающую роль. То же самое относится и к расходным материалам, используемым в процессе изготовления плат

Сложно разделить производственные процессы по величине вклада, вносимого каждым из них в итоговую надежность ПП. Однако качество выполнения некоторых из процессов, например такого как металлизация переходных отверстий, оказывает прямое влияние на долгое и успешное функционирование плат. Данный процесс состоит из трех 
операций: предварительная очистка внутренней поверхности отверстия от так называемых продуктов наволакивания - остатков смолы после сверления; предварительная химическая металлизация и, наконец, итоговая гальваническая металлизация, которая, в свою очередь, может осуществляться в несколько этапов. Для таких комплексных производственных процессов важен особый контроль всех параметров, а также промежуточный контроль результата, например измерение толщины итогового слоя меди в отверстиях до отправки заготовок плат на следующий процесс - формирование рисунка внешних слоев. Контроль промежуточного результата позволяет своевременно обнаружить любые отклонения и не допустить появления несоответствий на дальнейших этапах.

\section{ВЫХОДНОЙ КОНТРОЛЬ И ИСПЫТАНИЯ}

В ходе эксплуатации конечного изделия входящая в его состав плата будет испытывать различные неблагоприятные воздействия. То, как долго ПП будет успешно функционировать, напрямую связано с сохранением ею структурной целостности, то есть с тем, насколько технологичной была разработана конструкция, насколько качественно были выполнены процессы прессований и металлизаций, какие использовались материалы.

Прежде всего на структурную целостность негативно влияют циклы нагрева и охлаждения. Очевидно, что для большинства применений максимальный температурный стресс - это процесс пайки, ведь плата за короткий промежуток времени нагревается до температуры порядка $250^{\circ} \mathrm{C}$. В случае наличия штыревого монтажа или пайки волной ПП повторно подвергается температурному воздействию, которое является еще более жестким. Как убедиться, что надежность ПП достаточна и что после монтажа мы не получим «бомбу замедленного действия» в виде скрытых дефектов, готовых проявиться в любой момент? Убедиться можно, применяя метод изготовления так называемых микрошлифов после симуляции процесса монтажа. После общего

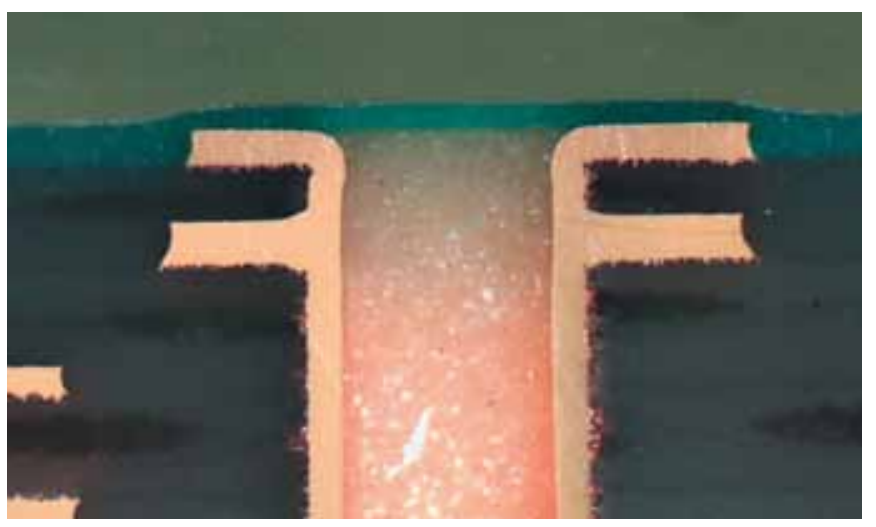

Рис. 3. Пример изображения микрошлифа, полученного с помощью микроскопа визуального контроля случайно выбранные печатные платы подвергают определенным температурным воздействиям. Для этого их подгружают в расплавленный припой согласно методикам, описанным в стандарте IPC-ТМ-650. Затем из платы вырезается небольшая часть так, чтобы в нее попали наиболее важныеэлементы, такиекакпереходные отверстия различных диаметров. Отделенный участок печатной платы заливается эпоксидной смолой, и затем торец зашлифовывается. Используя микроскоп, можно подробно изучить внутреннее строение ПП и оценить, насколько благополучно она перенесла температурное воздействие (рис. 3).

С помощью этого метода можно провести измерения толщин меди, межслойных расстояний, а также обнаружить отклонения, которые напрямую влияют на надежность и могут проявить себя далеко не сразу - спустя месяц, год или даже несколько лет. Например, на рис. 4 показан отрыв внутренней металлизации отверстия от прилегающего к нему внутреннего слоя, произошедший после симуляции процесса монтажа.

чаще всего причиной такого дефекта является некачественное выполнение очистки внутренней поверхности отверстия от продуктов наволакивания перед химической металлизацией. Но, как отмечалось ранее, неправильная конструкция платы также может привести к появлению таких нарушений структурной целостности.

\section{ПРЕДЪЯВЛЯЕМЫЕ ТРЕБОВАНИЯ}

Еще одна важная тема, касающаяся надежности печатных плат, - классы IPC. Как известно, их всего три. Выбирая тот или иной класс, мы сразу предъявляем требования к ПП, начиная с ее проекта. Напомним, что наиболее распространенным является класс 2, который соответствует требованиям надежности к самому большому диапазону конечных изделий и условиям их применения - промышленной электронике, средствам связи, измерительной технике, компьютерам и т.д. Класс 1 - это упрощенные требования, которые, как правило, относятся к бытовой технике. Наконец,

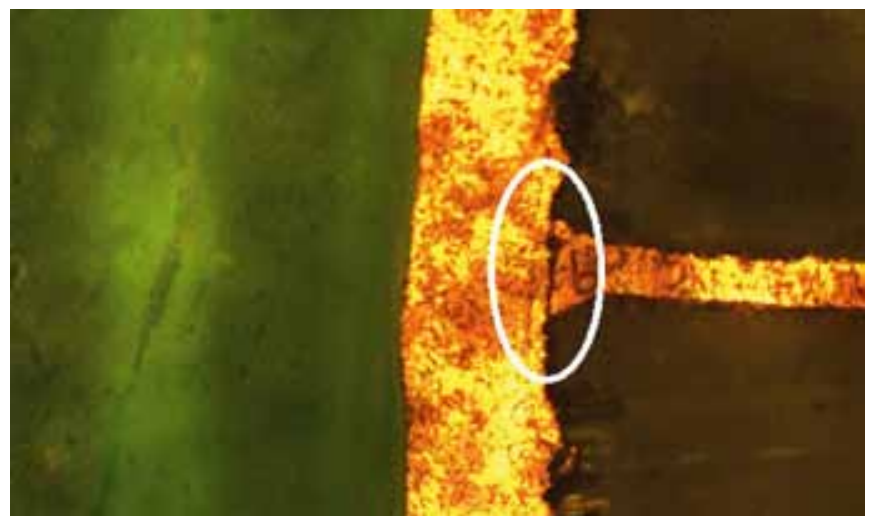

Рис. 4. Отрыв металлизации отверстия от внутреннего слоя 
класс 3 - это требования к надежности, которые применимы в тех случаях, когда отказы недопустимы, например аппараты ИвЛ, кардиостимуляторы, бортовая аппаратура летательных аппаратов, включая космические, и т.д.

Различия требований к параметрам ПП разных классов огромны. Будучи ограниченными в объеме статьи, коснемся лишь одного очень важного аспекта - верификации толщины металлизации переходных отверстий. Для класса 3 средняя толщина должна быть не менее 25 мкм. Но на плате могут быть сотни и даже тысячи отверстий, к тому же разных диаметров! Как подтвердить, что все они соответствуют критерию? К сожалению, на сегодняшний день наиболее распространенным и надежным методом контроля толщины металлизации остается изготовление микрошлифов. Данный метод относится к методам разрушающего контроля. В стандарте IPC-6012 подробно описано, как выбирать отверстия и направления реза (рис. 5).

Другой важный вопрос - сколько нужно изготовить дополнительных микрошлифов. Стандарт регламентирует это количество: его можно определить по таблице 4-2 в зависимости от объема партии и величины AQL (Acceptable Quality Level - приемлемый уровень качества), которая,

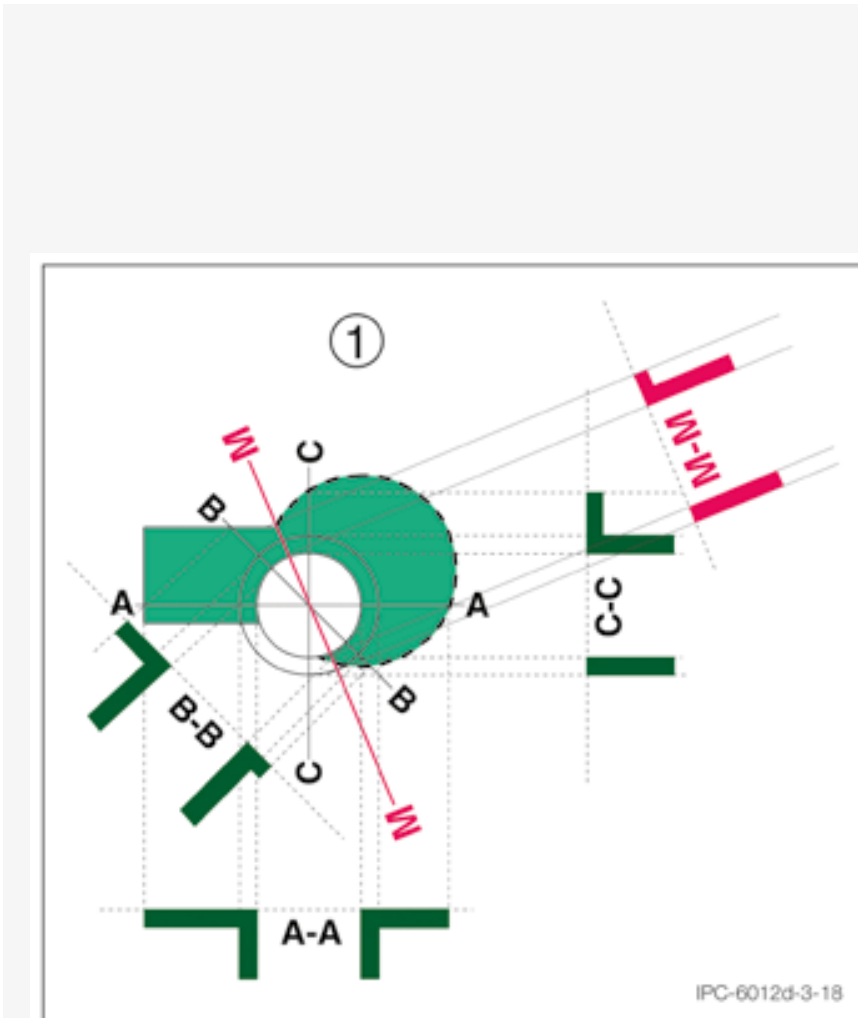

Figure 3-18 Microsection Rotations for Breakout Detection

Note 1. Only a vertical cut at M-M reveals the minimum conductor width. в свою очередь, определяется классом изделия и видом контролируемого параметра.

Для некоторых сочетаний AQL и объема партии количество дополнительных микрошлифов для верификации может быть сравнимо с объемом самой партии плат и даже превосходить его. Например, для верификации толщины металлизации отверстий при $\mathrm{AQL}=0,10$ и объеме партии от 91 до 150 панелей необходимо выполнить 125 микрошлифов. Если к этому добавить трудозатраты на сам процесс верификации, то становится очевидно, что себестоимость плат возрастает критически.

Компания NCAB предлагает компромиссный подход: мы даем потребителю на выбор три уровня (варианта) верификации:

- уровень 1 - полная верификация в соответствии со стандартом IPC-6012 класс 3;

- уровень 2 - при объеме партии более 25 панелей количество микрошлифов определяется по таблице 4-2 при AQL=4,0; если же партия не более 25 панелей, то верификация производится по двум шлифам;

- уровень 3 - контроль производится по двум шлифам, независимо от размера партии.

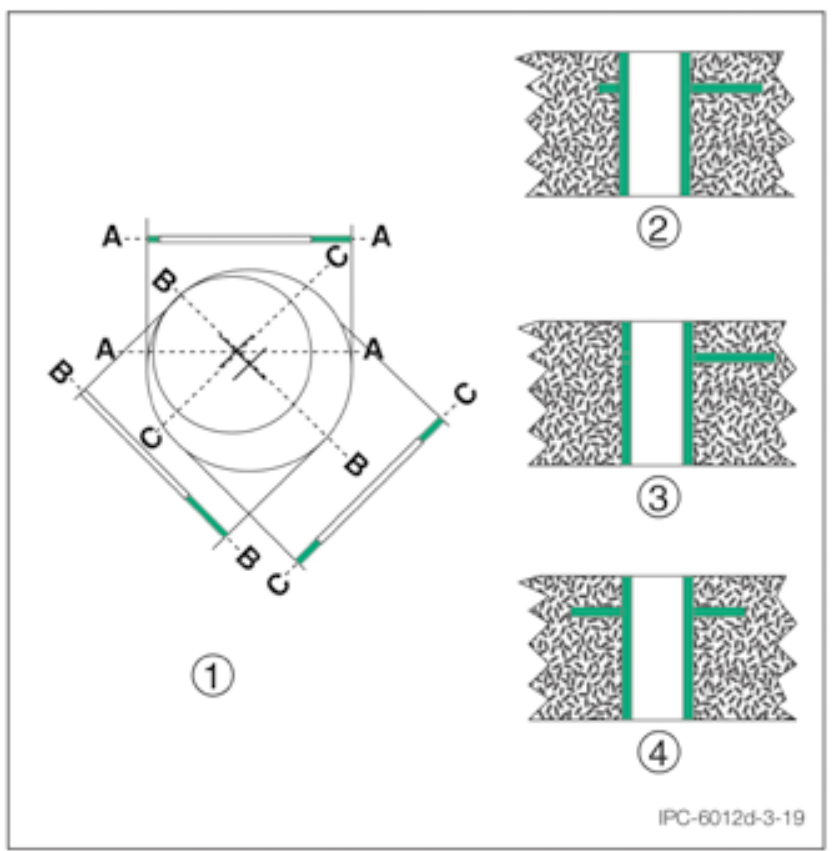

Figure 3-19 Comparison of Microsection Rotations

Note 1. Only microsection cut B-B reveals the actual annular ring reduction. Note 2. If the mount were cut through section A-A, the microsection would look like this.

Note 3. If the mount were cut through section B-B, the microsection would look like this.

Note 4. If the mount were cut through section C-C, the microsection would look like this.

Рис. 5. Примеры иллюстраций из стандарта IPC-6012D, указывающие направления реза при изготовлении микрошлифов. Использованы с разрешения ассоциащии IPC 
Таким образом, в дополнение к ПП наивысшего класса надежности мы предлагаем потребителю решения в виде нескольких промежуточных уровней верификации. Этот подход проверен и одобрен многими известными российскими и зарубежными компаниями

Стандарты ІРС содержат требования, выполнение которых предполагает, что мы ожидаем от ПП высокой надежности, соответствующей заявленному классу. Тем не менее, на протяжении многих лет работы, поставляя ПП на десятки рынков мира, мы сталкивались с тем, что стандарты не учитывают ряд нюансов. Мы получали обратную связь от потребителей, которые сообщали о том, что в некоторых конкретных случаях ПП, соответствующие требованиям стандартов IPC, не удовлетворяют заказчика. Результатом рассмотрения подобных ситуаций стало создание собственной спецификации NCAB, расширяющей и дополняющей стандарты IPC. На сегодняшний день наша спецификация включает 103 различных требования, которым следуем мы и наши партнерские производства. Причем это живой документ, который изменяется и развивается.

Рассмотрим примеры этих дополнительных требований.

\section{Пример 1. Горячее лужение (HAL)}

Горячее лужение является одним из наиболее распространенных финишных покрытий, обеспечивающим отличную паяемость. К тому же это и одно из самых дешевых покрытий. Казалось бы, какие риски могут возникать в хорошо отработанной технологии?

Дело в том, что сувеличением плотности монтажа стало назревать противоречие между требованиями некоторых потребителей и стандарта IPC-6012. Сегодня всем понятно, что для поверхностного монтажа корпусов с шагом менее 0,5 мм необходимо использовать иммерсионные покрытия типа ENIG, ImAg и т. п., которые обеспечивают высокую степень плоскостности поверхности контактных площадок. Но на начальном этапе «SMT-революции» не все производители электронного оборудования могли отказаться от отработанной технологии горячего лужения по разным причинам. Изучив требования различного оборудования поверхностного монтажа и возможности настройки машин горячего лужения, наши эксперты добавили ктребованиям IPC-6012 то, что толщина горячего лужения должна находиться в диапазоне от 1 до 40 мкм.

Таким образом, ужесточив требования к своей продукции, NCAB позволил своим потребителям расширить сферуприменения горячего лужения и, следовательно, снизить себестоимость изделий.

\section{Пример 2. Толщина металлизации переходных отверстий}

C 2006 года директива RoHS ограничивает применение тяжелых металлов, в том числе свинца, в электронных изделиях. С точки зрения процесса монтажа самое существенное изменение, вызванное отсутствием свинца в припое, - повышенная температура пайки. Появление бессвинцой пайки заставило нас доработать внутренний стандарт NCAB. Дело в том, что коэффициент теплового расширения (КТР) стандартных материалов типа FR-4 в несколько раз выше, чем КТР меди. Во время высокотемпературной пайки материал настолько расширяется, что может буквально разорвать столбики металлизации переходных отверстий или по крайней мере сильно их деформировать. Результатом этого является неприятный и опасный дефект - «плавающий» контакт. Выход из данной ситуации очевиден - применение высокотемпературных материалов (например, FR-4 HiTg), которые сохраняют свою геометрию в широком температурном диапазоне. Однако использование материалов среднего температурного диапазона все же может спровоцировать описанный выше дефект.

Наши специалисты предположили, что риски нарушения структурной целостности столбиков металлизации переходных отверстий можно существенно снизить, увеличив толщину меди на стенках отверстий. В ходе экспериментов совместно с одним из европейских контрактных производителей выяснилось, что при увеличении толщины металлизации с 20 мкм (в соответствии с IPC-6012D класс 2) до 25 мкм (требование IPC-6012D класс 3) надежность существенно повышается. Нами было решено добавить требование к толщине металлизации в 25 мкм по умолчанию для всех наших ПП. То есть ПП, изготовленные по IPC-6012D класс 2 с учетом требований нашей спецификации, отвечают повышенным требованиям к надежности межслойных соединений.

Кроме рассмотренных примеров, спецификация NCAB учитывает множество других аспектов, повышающих надежность ПП. Это и ограничение спектра используемых материалов (в соответствии с опытом их применения), и использование надежных технологий с запретом альтернативных (например, гибко-жесткие платы производятся только по безадгезивной технологии, обеспечивающей лучшую структурную целостность, и ни в коем случае не по адгезивной), и запрет на ремонт разрывов проводников, и даже косметические требования к внешнему виду ПП (отсутствие царапин и т. п.). При изготовлении печатных плат мы закладываем все эти требования по умолчанию, то есть потребитель при заказе об этом может и не знать. Но в итоге это позитивно влияет на надежность как ПП, так и конечного продукта

Наш многолетний опыт показывает, что применение описанных подходов позволяет повысить надежность печатных плат, а следовательно, и конечных электронных устройств. Это также подтверждают наши потребители, работающие на многих рынках мира. 


\section{Потому что отказы недопустимы}

Электроника окружает нас в повседневной жизни. Автомобиль, поезд, самолет, аппарат ИВЛ или кардиостимулятор - мы полагаемся на их надежность. Мы ожидаем, что эти устройства будут работать - потому что это критически важно.

Все электронные устройства собираются на печатных платах. Может показаться, что все печатные платы выглядят одинаково. Однако между "стандартными" печатными платами и платами с высокой надежностью имеются колоссальные различия. Именно в деталях кроется разница. Начиная с дизайна, правильной спецификации, выбора подходящего партнера для производства, и заканчивая логистикой, точностью поставок и подходом ко всему производственному процессу - все имеет значение.

Потому что отказы недопустимы.

Печатные платы с высокой надежностью. 